\title{
ESTUDIO PARA LA DETECCIÓN DE LAS NECESIDADES DE EXTENSIÓN Y SERVICIOS HACIA LA SOCIEDAD CON BASE EN LOS OBJETIVOS DE DESARROLLO SOSTENIBLE
}

\section{STUDY FOR THE DETECTION OF THE NEEDS OF EXTENSION AND SERVICES TOWARDS THE SOCIETY BASED ON THE SUSTAINABLE DEVELOPMENT GOALS}

\section{Sebastián Reyes Alvarado ${ }^{1}$ \\ Universidad Santander, Panamá}

\section{RESUMEN}

El firme compromiso con la visión de país para el logro de los Objetivos de Desarrollo Sostenible

$1 \quad$ Doctor en Educación y Vicerrector de Investigación y Extensión de la Universidad Santander, Panamá. Afiliación de REDIPE N²014.

$N^{\circ}$ ORCID: https://orcid.org/0000-0002-5824-9832

Google académico: https://bit.ly/38/sYMv

vicerrectoria@usantander.edu.pa (507) 394-3470 ha permitido que la Universidad Santander de Panamá, a través, de este estudio, constituyera una la línea base articuladora que sustenta el accionar y servicio hacia la sociedad, tomando en consideración las principales necesidades en materia de extensión, lo que permitirá, desde la gestión de proyectos, programas, campañas, talleres, trabajos comunitarios, cursos y otras actividades de carácter académico e 
investigativo, el afianzamiento de la filosofía universitaria con perspectiva ética, sustentando así, el trabajo y aporte que generarían los factores de extensión, investigación, gestión y academia en beneficio del desarrollo social y comunitario.

PALABRAS CLAVE: Extensión universitaria, Objetivo de Desarrollo Sostenible, Sociedad.

\section{ABSTRACT}

The firm commitment to the vision of the country for the achievement of the Sustainable Development Goals has allowed the Santander University of Panama, through this study, to constitute an articulating baseline that supports the actions and service towards society, taking into account the main needs in terms of extension, which will allow, from the management of projects, programs, campaigns, workshops, community work, courses and other academic and research activities, the strengthening of university philosophy with an ethical perspective, thus supporting the work and contribution that the extension, research, management and academy factors would generate for the benefit of social and community development.

\section{KEYWORDS:}

University extension, Sustainable Development Goals, Society

\section{INTRODUCCIÓN}

La determinación de necesidades a nivel comunitario es fundamental para lograr procesos de intervención que coadyuven a mejorar las realidades que se ven dentro de las mismas; no obstante, para que esto sea exitoso se ha de requerir la participación de los diversos actores, a fin de que aporten en la determinación de sus falencias y a la vez en la generación de posibles soluciones.
En esta dirección el estudio se centra en la determinación de aquellas necesidades que en materia de extensión surgen dentro de las comunidades, tomando como base aquellas realidades que son prioritarias y que muchas veces han sido desatendidas o que las acciones realizadas al no ser prioritarias o consensuadas, no generan el impacto respectivo. Poder diagnosticar estas necesidades, brindará la posibilidad de encaminar los servicios que requieren las propias comunidades, especialmente de la provincia de Panamá, Panamá Oeste y áreas aledañas, las cuales mantienen diversas realidades estructuradas de acuerdo a su condición económica, social, laboral, educativa, entre otros, a su vez, es un área con una densidad demográfica muy alta.

De llegar a establecerse esta línea base, en dónde los proyectos educativos a nivel de intervención puedan sustentarse y encontrar cabida, será mucho mayor el impacto que se pueda generar a partir de las acciones que se emanen de esta casa de estudio.

\section{MARCO TEÓRICO}

\section{Objetivos de Desarrollo Sostenible}

Los Objetivos de Desarrollo Sostenible se gestaron en la Conferencia de las Naciones Unidas sobre el Desarrollo Sostenible, celebrada en Río de Janeiro en 2012. El propósito era crear un conjunto de objetivos mundiales relacionados con los desafíos ambientales, políticos y económicos con que se enfrenta nuestro mundo.

Panamá se unió el 25 de septiembre de 2015 a los países que se comprometieron en la Asamblea General de las Naciones Unidas a orientar sus políticas y programas al logro de los 17 Objetivos de Desarrollo Sostenible antes del 2030.

A partir de este compromiso, Panamá ha asumido los Objetivos de Desarrollo Sostenible como 
pauta para guiar los esfuerzos de desarrollo y ha iniciado un proceso de apropiación nacional mediante el fortalecimiento del vínculo entre el Gobierno Nacional, los ciudadanos, y las organizaciones de la sociedad civil. Este proceso ha resultado en avances normativos, institucionales, operativos y de seguimiento, para garantizar que los esfuerzos del país se orienten hacia el cumplimiento de los Objetivos de Desarrollo Sostenible (Gobierno de la República de Panamá, 2017, pág. 4).

Cabe señalar, que según las Naciones Unidas son 17 los Objetivos de Desarrollo Sostenible, los cuales son:

1. Fin de la pobreza

2. Hambre cero

3. Salud y Bienestar

4. Educación de calidad

5. Igualdad de género

6. Agua limpia y saneamiento

7. Energía asequible y no contaminante

8. Trabajo decente y crecimiento económico

9. Industria, innovación e infraestructura

10. Reducción de las desigualdades

11. Ciudades y comunidades sostenibles

12. Producción y consumo responsable

13. Acción por el clima

14. Vida submarina

15. Vida de ecosistemas terrestres

16. Paz, justicia e instituciones sólidas

17. Alianzas para lograr los objetivos (Organización de las Naciones Unidas, 2015).

\section{Marco legal}

Dentro de la perspectiva legal podemos enunciar que las universidades tanto estatales como particulares, tienen que pasar por el proceso de evaluación institucional para su acreditación a cargo del Consejo Técnico de Fiscalización, en dónde es de estricto cumplimiento una serie de indicadores tal como lo establece el Decreto Ejecutivo 539 de 30 de agosto de 2018, en su artículo 2 el cual destaca que "El Sistema Nacional de Evaluación y Acreditación para el Mejoramiento de la Calidad de la Educación Superior Universitaria de Panamá, comprende el conjunto de organismos, normas y procedimientos estructurados e integrados funcionalmente, según lo establece el artículo 2 de la Ley $N^{\circ} 52$ de 26 de junio de 2015, sujeto a la orientación y políticas generales del Estado, cuya finalidad es establecer los criterios, indicadores y estándares para asegurar la calidad de la educación superior universitaria del país" (Ministerio de Educación, 2018, pág. $3)$.

Por otro lado, el presente estudio se ha fundamentado en el compromiso adquirido por parte del Estado panameño según Decreto Ejecutivo 393 del 14 de septiembre 2015, que adopta los objetivos de desarrollo sostenible y dicta otras disposiciones, el cual, en su artículo 1, establece "se adopta los objetivos de desarrollo sostenible como una pauta para el desarrollo del gobierno de la República de Panamá" (Ministerio de Relaciones Exteriores, 2015, pág. 3).

Cabe señalar, que esta adopción de los objetivos de desarrollo sostenible se ve reforzada cuando se establece el Decreto Ejecutivo $\mathrm{N}^{\circ} 75$ de viernes 29 de junio de 2018, Que declara el mes de julio, mes de los objetivos de desarrollo sostenible, dentro del cual, en su artículo 1 enuncia "Se establece el mes de julio como mes de los objetivos de desarrollo sostenible para la realización de actividades de divulgación, académicas, culturales, artísticas, ambientales 
y sociales a nivel nacional" (Ministerio de Desarrollo Social, 2018, pág. 3).

Por otro lado, en su artículo 4, del mencionado Decreto Ejecutivo $N^{\circ} 75$ "Se solicita a todas las organizaciones no gubernamentales, asociaciones, empresa privada, organismos internacionales, clubes cívicos y la comunidad en general, que participen de forma activa del mes de los Objetivos de Desarrollo Sostenible" (Ministerio de Desarrollo Social, 2018, pág. 3).

\section{METODOLOGÍA}

El presente estudio se base en el paradigma cualitativo, partiendo de un diseño etnográfico, dado que se trabajó con un grupo representativo de la sociedad, a su vez, se utilizó el método de revisión documental para recabar datos contenidos en informes, publicaciones y/u otros elementos de carácter bibliográfico y oficiales (Bernal, 2010, págs. 111-112).

\section{PARTICIPANTES}

La población objeto del presente estudio, estuvo conformada, por diversos integrantes de la sociedad, desde estudiantes, profesionales y hasta líderes comunitarios. A su vez, se realizó una búsqueda de los documentos o elementos bibliográficos que aportasen información actualizada que sirviera de base para la construcción de este estudio.

Es por ello, que el tipo de muestreo a utilizar fue el de participantes voluntarios o autoseleccionados, ya que las personas fueron propuestas para participar del estudio, solicitando para ello, su anuencia como participantes, a través de una invitación formal y previamente realizada (Hernández Sampieri, Fernández Collado, \& Baptista Lucio, 2014, págs. 386-387), además, se levantó un inventario de los documentos que se iban a revisar para la parte documental.

Por otro lado, el total de participantes fue de 16 personas, los cuales, de manera voluntaria accedieron a ser parte del estudio, paro lo cual, se segmentaron en 4 grupos, quedando la distribución de la siguiente manera:

- Un grupo de 4 docentes y estudiantes universitarios.

- Un grupo de 4 maestros de educación básica general.

- Un grupo de 4 estudiantes de educación básica general.

- Un grupo de 4 líderes sociales.

Cabe señalar, que esta actividad fue desarrollada en el Distrito de La Chorrera, no obstante, las personas que fueron participes del estudio, son representativas de diversas áreas, algunas de ellas viven y trabajan en la provincia de Panamá y otras en provincia de Panamá Oeste. Lo cual, permitió tener una visión mucho más amplia de las realidades comunitarias.

Por otro lado, se utilizó como punto de encuentro y desarrollo de las acciones la Biblioteca Pública Hortensio de Icaza, de La Chorrera, Panamá Oeste, dado que se encuentra en la comunidad y fue cedido para esta actividad de manera gratuita.

\section{PROCEDIMIENTO PARA LA RECOLECCIÓN DE DATOS}

La recolección de datos se llevó a cabo en diversas etapas, las cuales son:

- Etapa 1: revisión documental: en esta etapa, se procedió a revisar estudios, investigaciones, documentos oficiales, normativas $u$ otros referentes bibliográficos, nacionales e internacionales, que permitiesen conocer las problemáticas de la comunidad, cuyos resultados serían contrastado con lo obtenido en las demás etapas. 
- Etapa 2: sensibilización: En esta etapa, se explicaron las bases del estudio, los fines que se perseguían y la importancia del estudio, a su vez, se les dio una presentación acerca de los Objetivos de Desarrollo Sostenible, de manera que estuviesen contextualizados con respecto a la temática y pudiesen pasar muchos más claros a las otras etapas.

- Etapa 3: Taller: En esta etapa, se desarrolló un taller, tomando como base los Objetivos de Desarrollo Sostenible, afín de que los participantes, identificaran desde sus perspectivas comunitarias y sociales, las principales dificultades que se presentan, de cara al cumplimiento de los Objetivos de Desarrollo Sostenible.

- Etapa 4: Estudio Focal: en esta etapa, a través del proceso de entrevista grupal, se visualizaron las acciones, sugerencias, alternativas o propuestas de mejora, dadas por los diversos actores, las cuales, integran los Objetivos de Desarrollo Sostenible y que permiten la articulación y el accionar de la universidad, desde diversas áreas.

\section{ANÁLISIS DE LOS DATOS}

En primera instancia se procedió a analizar los hallazgos encontrados de la revisión documental, lo que permitió poner en contexto algunas realidades o problemáticas que se están dando en comunidad y que serían objeto del presente estudio.

Posteriormente se desarrolló el análisis de resultados del taller, el mismo, se dio mediante el cruce de variables, para lo cual, se utilizó como referencia una espina de pescado que permite una mejor visualización de los hallazgos.

Por último, tomando como referencia los hallazgos anteriores y los resultados del estudio focal, se procedió a cruzar dicha información con lo resultante del estudio focal, para levantar una línea base articuladora, de todas las posibles acciones que, desde el interior de la universidad, se pueden implementar para responder a estas problemáticas.

\section{RESULTADOS}

A continuación, se detallan los resultados más relevantes del estudio. Una vez finalizada la revisión documental, se arrojaron los siguientes resultados:

En Panamá "entre 2013 y 2016, las incidencias de pobreza general y pobreza extrema a nivel nacional disminuyeron respectivamente de $26.2 \%$ a $22.1 \%$, y de $11.1 \%$ a $9.9 \% .15$. Sin embargo, al analizar esta información se evidencian brechas entre las áreas urbanas y rurales. En áreas rurales, la incidencia de pobreza general y pobreza extrema en 2016 fue de $45.2 \%$ y $24.8 \%$, contra $11.1 \%$ y $2.8 \%$ en áreas urbanas. En el caso de los hogares en pobreza general este aumento fue de $6.3 \%$ $y$ en los de extrema $17.1 \%$. Por otro lado, según el Índice de Pobreza Multidimensional de Panamá que permite conocer tanto la incidencia como intensidad de la pobreza, analizando dimensiones como educación, trabajo, salud; vivienda, servicios básicos y acceso a Internet; y ambiente, entorno y saneamiento. Al estudiar las carencias que padecen los hogares, según está primera medición, las cuatro de las cinco dimensiones aportan casi en igual medida, alrededor del $20 \%$ a la pobreza multidimensional, Educación (23.9\%), Trabajo (20.9\%), Ambiente, entorno y saneamiento (20.7\%) y Vivienda y servicios básicos (19.8\%). En el caso de salud, la misma solamente contribuye un $14.7 \%$ a la pobreza multidimensional en los hogares" (Gobierno de la República de Panamá, 2017, págs. 35-37).

Luego de obtener estos resultados, se puede observar que los temas de pobreza, hambre, 
salud, educación, vivienda y bienestar, medio ambiente y saneamiento y trabajo, fueron los más sensitivo, de acuerdo a cifras manejadas por el Estado panameño, lo que se conecta de manera directa con los Objetivos de Desarrollo Sostenible.

Cabe señalar, que en la tabla 1 , se aprecia la triangulación de los resultados del trabajo realizado en los talleres, con base en los Objetivos de Desarrollo Sostenible, en dónde, a través de un cruce de variables, se pudo determinar los aspectos más relevantes manifestados por la comunidad y de los cuales, fueron de mayor repetición entre los grupos. Con estos aportes, se pudo determinar dónde estaban los mayores problemas de la comunidad y como estos se entrelazaban con los Objetivos de Desarrollo Sostenible.

Tabla 1.

Triangulación de los resultados de los talleres

\begin{tabular}{|c|c|}
\hline Objetivo de desarrollo Sostenible & Cruce de variables \\
\hline Objetivo 1: Fin de la pobreza & $\begin{array}{l}\text { - Educación. } \\
\text { - Desigualdad } \\
\text { - Salud }\end{array}$ \\
\hline Objetivo 2: Hambre cero & $\begin{array}{l}\text { - Innovación agrícola y conservación de los recursos } \\
\text { naturales(sostenibilidad) } \\
\text { - Desigualdad } \\
\text { - educación } \\
\end{array}$ \\
\hline Objetivo 3: Salud y bienestar & $\begin{array}{l}\text { - Pertinencia en las políticas publicas } \\
\text { - Educación }\end{array}$ \\
\hline Objetivo 4: Educación de calidad & $\begin{array}{l}\text { - Pertinencia en políticas públicas (edilicios) } \\
\text { - Educación orientada a desarrollar competencias y } \\
\text { talentos. } \\
\text { - Desigualdad }\end{array}$ \\
\hline Objetivo 5: Igualdad de género & $\begin{array}{ll}\text { - } & \text { Desigualdad } \\
\text { - } & \text { Pertinencia de las políticas publicas } \\
\text { - } & \text { Paradigmas culturales } \\
\end{array}$ \\
\hline Objetivo 6: agua limpia y saneamiento & $\begin{array}{l}\text { - } \text { Pertinencia de las políticas publicas } \\
\text { - Educación } \\
\text { - Paradigmas culturales } \\
\text { - Salud }\end{array}$ \\
\hline
\end{tabular}




\begin{tabular}{|c|c|}
\hline Objetivo 7: Energía renovable & $\begin{array}{l}\text { - Pertinencia de las políticas publicas } \\
\text { - Corrupción } \\
\text { - Economía } \\
\text { - Educación } \\
\text { - Conciencia ambiental } \\
\text { - Poca investigación }\end{array}$ \\
\hline $\begin{array}{l}\text { Objetivo 8: Empleo digno y crecimiento } \\
\text { económico. }\end{array}$ & $\begin{array}{l}\text { - Pertinencia de las políticas publicas } \\
\text { - Desigualdad } \\
\text { - Corrupción } \\
\text { - Pertinencia de las económicas }\end{array}$ \\
\hline $\begin{array}{l}\text { Objetivo 9: Industria innovación } \\
\text { infraestructura }\end{array}$ & $\begin{array}{l}\text { - } \\
\text { - Poca investigación } \\
\text { - } \text { Corrinencia de las políticas publicas } \\
\text { - Cooperación interinstitucional } \\
\text { - Pertinencia de las económicas } \\
\end{array}$ \\
\hline Objetivo 10: Reducción de las desigualdades & $\begin{array}{ll}\text { - } & \text { Educación } \\
\text { - } & \text { Pertinencia de las políticas publicas } \\
\text { - } & \text { Falta de cultura en ética valores (equidad) } \\
\end{array}$ \\
\hline $\begin{array}{l}\text { Objetivo 11: Ciudades y comunidades } \\
\text { sostenibles }\end{array}$ & $\begin{array}{l}\text { - } \\
\text { - Eertinencia de las políticas publicas } \\
\text { - } \text { Falta de cultura en sostenibilidad } \\
\text { - Edilicios } \\
\text { - Educación continua de la gestión sostenibilidad }\end{array}$ \\
\hline $\begin{array}{l}\text { Objetivo 12: Producción y consumo } \\
\text { responsable }\end{array}$ & $\begin{array}{ll}\text { - } & \text { Consumismo } \\
\text { - } & \text { Investigación responsable } \\
\text { - } & \text { Pertinencia de las políticas publicas } \\
\text { - } & \text { Educación } \\
\end{array}$ \\
\hline Objetivo 13: Acción por el clima & $\begin{array}{l}\text { - Pertinencia de las políticas publicas } \\
\text { - Poca divulgación y aplicación en normativas } \\
\text { - Educación } \\
\text { - Poca conciencia ambiental }\end{array}$ \\
\hline
\end{tabular}




\begin{tabular}{|c|c|}
\hline Objetivo 14: Vida submarina & $\begin{array}{l}\text { - Pertinencia de las políticas publicas } \\
\text { - Poca investigación científica y técnica de la } \\
\text { contaminación submarina } \\
\text { - Poca conciencia ambiental } \\
\text { - La contaminación de los ríos y desagües }\end{array}$ \\
\hline Objetivo 15: Vida de ecosistemas terrestre & $\begin{array}{l}\text { - } \text { Regulación } \\
\text { - } \text { Pertinencia de las políticas publicas } \\
\text { - } \text { Poca divulgación y aplicación en normativas } \\
\text { - } \\
\text { - } \\
\text { Pducación } \\
\end{array}$ \\
\hline $\begin{array}{l}\text { Objetivo 16: Paz, justicia e instituciones } \\
\text { fuertes }\end{array}$ & $\begin{array}{l}\text { - } \text { Falta de cultura en ética valores (equidad) } \\
\text { - Pertinencia de las políticas publicas } \\
\text { - Poca divulgación y aplicación en normativas } \\
\text { - Corrupción } \\
\text { - Programas de educación para la paz en las } \\
\text { comunidades } \\
\text { - Burocracia }\end{array}$ \\
\hline Objetivo 17: Alianza para lograr los objetivos & $\begin{array}{l}\text { - } \text { Falta de información. } \\
\text { - } \quad \text { Falta de comunicación. } \\
\text { - } \quad \text { Faltan alianzas con otros países para lograr los } \\
\text { objetivos } \\
\text { - } \quad \text { Red de apoyo entre las autoridades, regionales, } \\
\text { autoridades locales e internacionales y comunidad } \\
\text { - Pertinencia de las políticas publicas } \\
\text { - Poca divulgación y aplicación en normativas }\end{array}$ \\
\hline
\end{tabular}

Fuente: elaboración a partir de los resultados obtenidos de los talleres y su cruce con los Objetivos de Desarrollo Sostenible.

Por otro lado, en la figura 1 , se aprecia el contraste entre los aspectos más relevantes obtenidos de los resultados del estudio focal y los Objetivos de Desarrollo Sostenible, para lo cual, se ha generado una matriz, esquematizando una espina de pescado, lo que ha permitido elementos que requieren ser abordados con prioridad.

En dicha matriz, se logró identificar aquellos aspectos que son los más vulnerables para las comunidades. 


\section{Figura 1.}

Matriz de tabulación de los resultados de los grupos focales

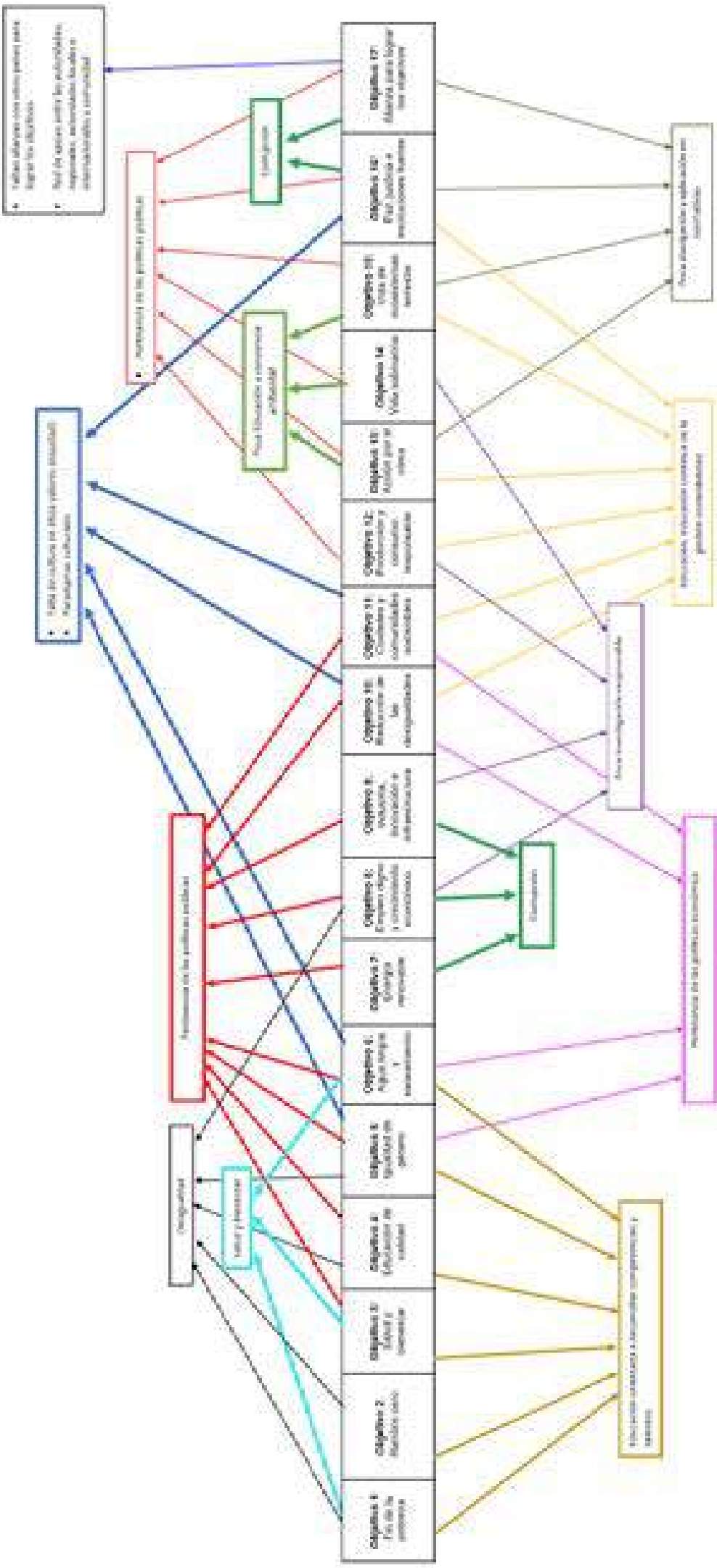

Fuente: elaboración en base a los resultados de los grupos focales en contraste con los Objetivos de Desarrollo Sostenible. 
Cabe señalar, que en la tabla 2, luego de contrastar los resultados de la revisión documental y lo aportado por los participantes de los talleres y grupos focales, se pudo generar una línea base articuladora y temática para la constitución de las diversas acciones que se emanaran desde las perspectivas de los factores universitarios y que responden a dichas dificultades comunitarias.

Tabla 2

Líneas o acciones a desarrollar por los diversos factores universitarios en base a los resultados de la revisión documental y el estudio focal.

\begin{tabular}{|c|c|}
\hline OBJETIVO & LÍNEAS/ACCIONES \\
\hline Objetivo 1: Fin de la pobreza & 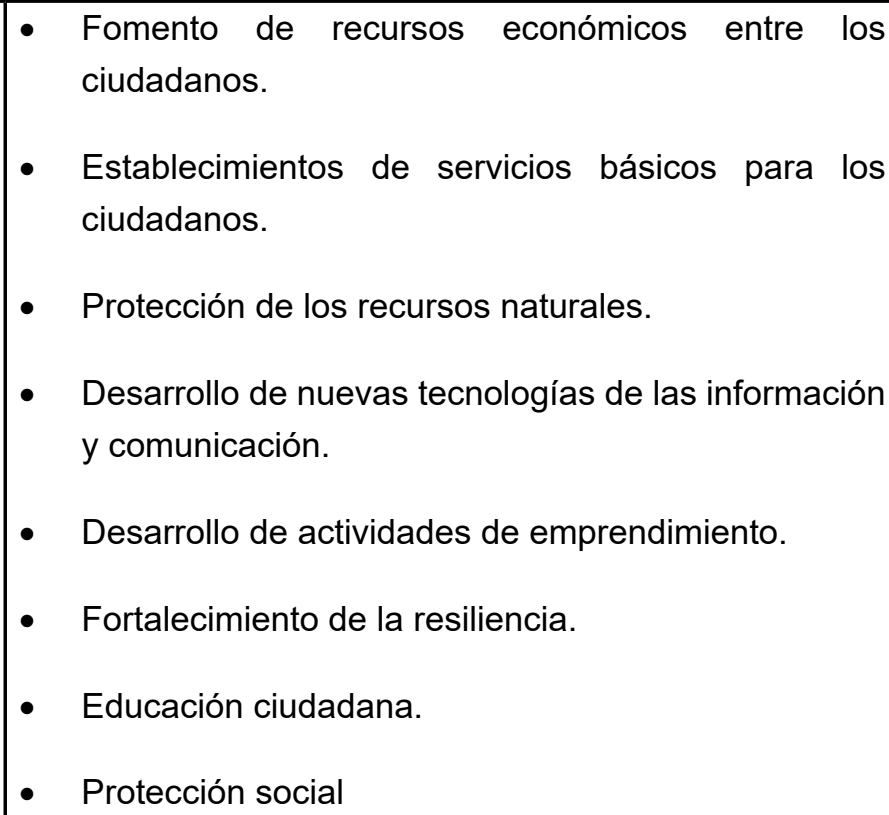 \\
\hline Objetivo 2: Hambre cero & $\begin{array}{l}\text { - Fomento de programas para el desarrollo de una } \\
\text { alimentación sana, nutritiva y suficiente. } \\
\text { - Promoción de la actividad agrícola en centros } \\
\text { educativos o comunidades vulnerables, a través de } \\
\text { la constitución o desarrollo de huertos, siembras } \\
\text { hidropónicos o ecológicos. } \\
\text { - Formación en nuevas tecnologías para la agricultura } \\
\text { y la generación de alimentos. } \\
\text { - Programas para el desarrollo sustentable de los } \\
\text { recursos naturales. } \\
\text { Fomento de investigaciones a nivel del medio } \\
\text { ambiente entre los estudiantes de diversos niveles } \\
\text { académicos. }\end{array}$ \\
\hline
\end{tabular}




\begin{tabular}{|c|c|}
\hline Objetivo 3: Salud y bienestar & $\begin{array}{l}\text { - Programas de prevención de las principales } \\
\text { enfermedades que aquejan a la población y que } \\
\text { ocasionan altos índices de muertes como, por } \\
\text { ejemplo: Diabetes mellitus, Cáncer, VIH, Hipertensión } \\
\text { arterial, Obesidad, Dislipidemias (colesterol, } \\
\text { triglicéridos, colesterol HDL y LDL), Enfermedades } \\
\text { renales, Dengue, entre otras. } \\
\text { - Programas de prevención sobre accidentes de } \\
\text { tránsito, de trabajo entre otros. } \\
\text { - Promoción de estilos o hábitos de vida saludable. } \\
\text { - Fomento de la educación para la salud. } \\
\text { Promoción de los controles prenatales en mujeres } \\
\text { sobre todo de áreas vulnerables. } \\
\text { - Campañas masivas de vacunación, dirigida a niños } \\
\text { y adultos mayores. } \\
\text { - Campañas de prevención en materia de salud } \\
\text { mental. } \\
\text { - Campañas de nutrición infantil. } \\
\text { campañas en materias de salud bucal. } \\
\text { condiciones de vulneración. } \\
\text { salud dirigido a personas con discapacidad. }\end{array}$ \\
\hline
\end{tabular}




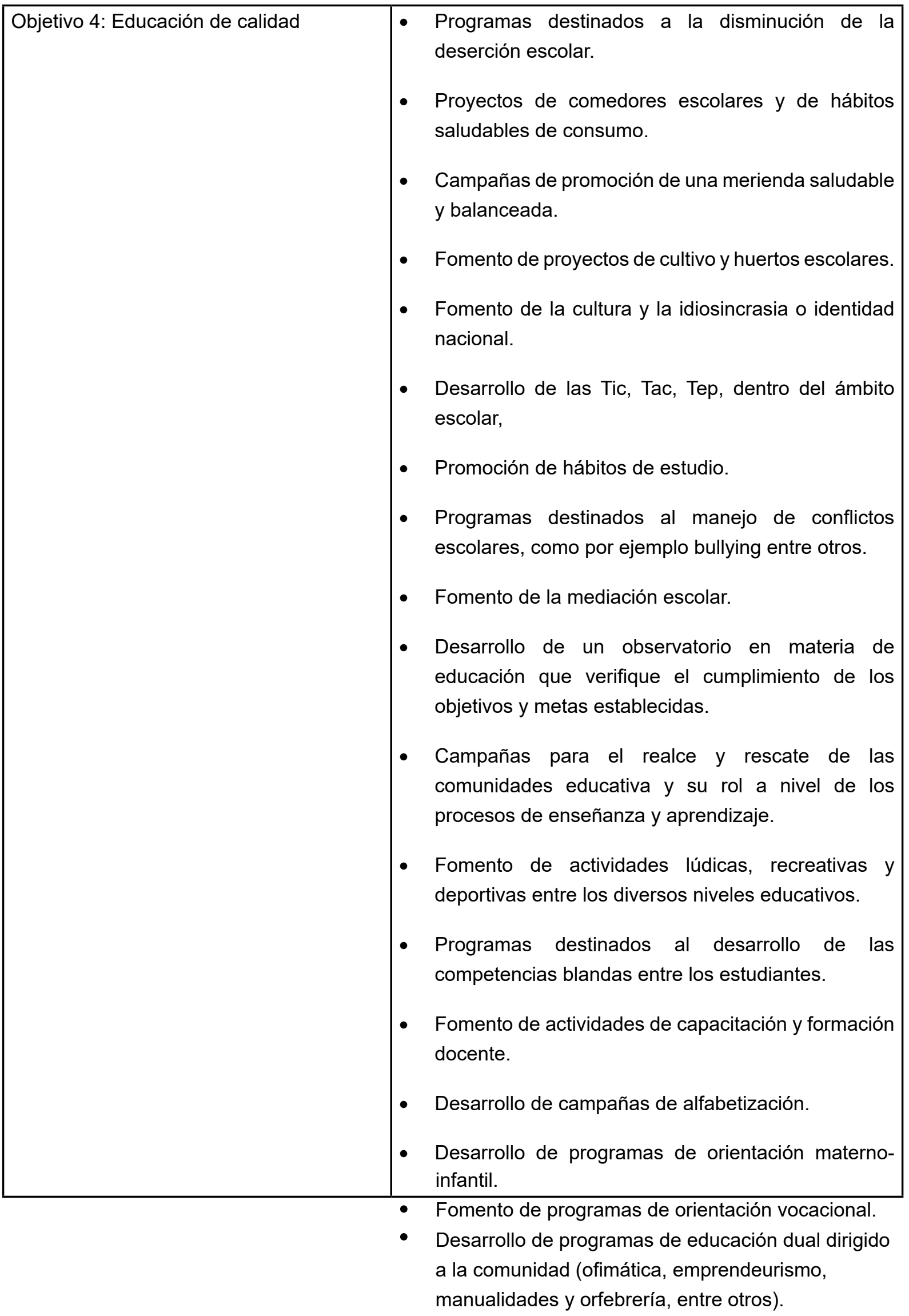




\begin{tabular}{|c|c|}
\hline Objetivo 5: Igualdad de género & $\begin{array}{l}\text { - Programas de prevención de actos de discriminación } \\
\text { hacia las mujeres y niñas, o hacia personas de } \\
\text { pensamiento, creencias o convicciones distintas. } \\
\text { - Fomento de campañas de promoción de igualdad de } \\
\text { oportunidades en el ámbito laboral y educativo. } \\
\text { - Programas de equiparación de oportunidades a las } \\
\text { mujeres en especial aquellas que padecen de alguna } \\
\text { condición de salud física o mental. } \\
\text { - Fomento del acceso universal a salud reproductiva } \\
\text { y sexual. } \\
\text { Campañas para promover los derechos igualitarios } \\
\text { en el acceso a recursos económicos, tierras y } \\
\text { propiedades. } \\
\text { Promoción de la paridad o igual de género en cargo } \\
\text { públicos o administrativos. }\end{array}$ \\
\hline
\end{tabular}




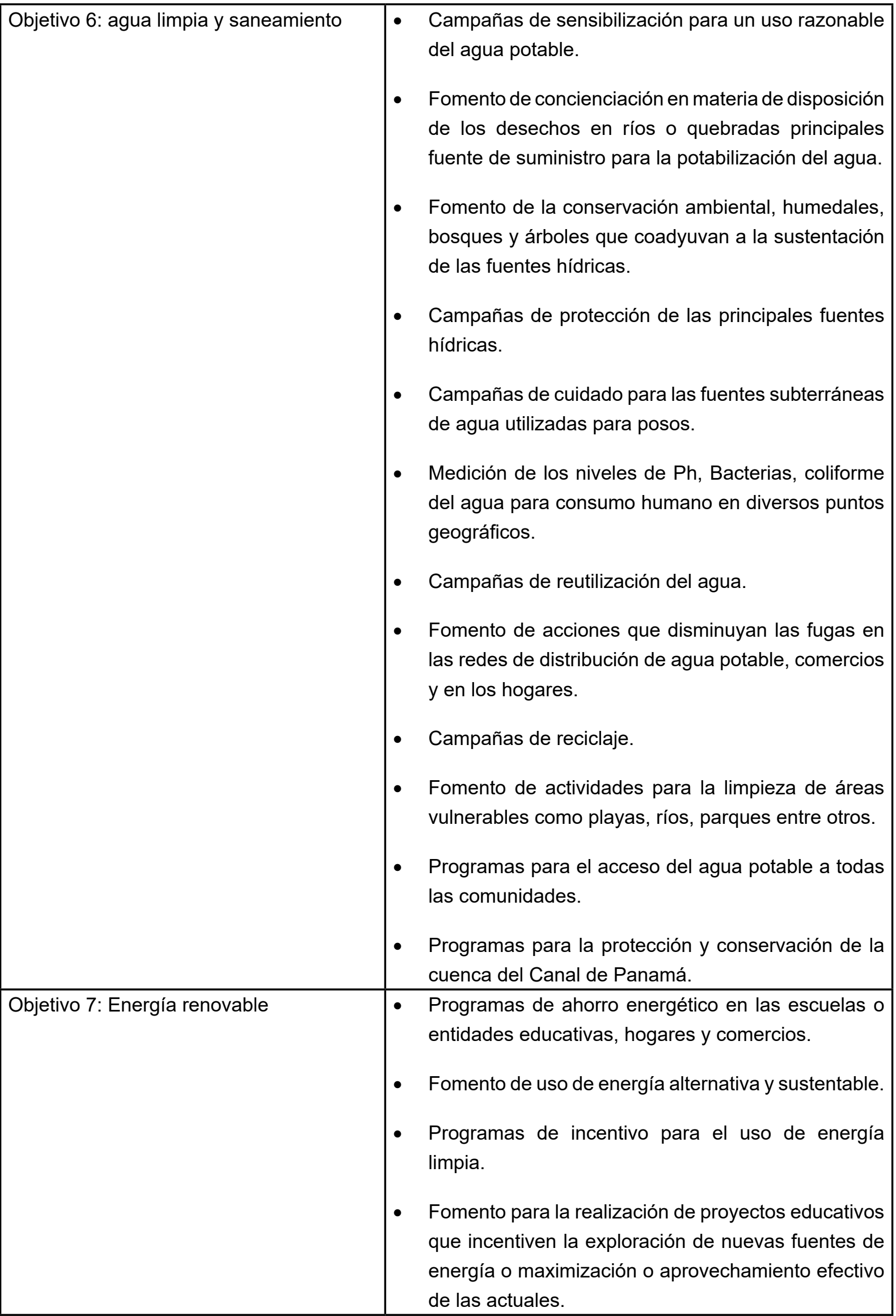




\begin{tabular}{|c|c|}
\hline $\begin{array}{l}\text { Objetivo 8: Empleo digno y crecimiento } \\
\text { económico. }\end{array}$ & $\begin{array}{l}\text { - Desarrollo de actividades que fomenten la } \\
\text { productividad. } \\
\text { - Desarrollo de acciones que incorporen el uso de } \\
\text { nuevas tecnologías de información y comunicación } \\
\text { al plano laboral. } \\
\text { - Fomento de espíritu emprendedor. } \\
\text { - Capacitación en materia laboral. } \\
\text { - Programas para la inserción de jóvenes al ámbito } \\
\text { laboral. } \\
\text { - Explicación de las leyes, decretos y normas en } \\
\text { materia laboral. } \\
\text { Fomentar la organización comunitaria para el } \\
\text { desarrollo de actividades económicas, a través de } \\
\text { cooperativas, organización o asociaciones. } \\
\text { - Promover acciones que fomente y fortalezca la } \\
\text { responsabilidad social corporativa. }\end{array}$ \\
\hline $\begin{array}{l}\text { Objetivo 9: Industria innovación e } \\
\text { infraestructura }\end{array}$ & 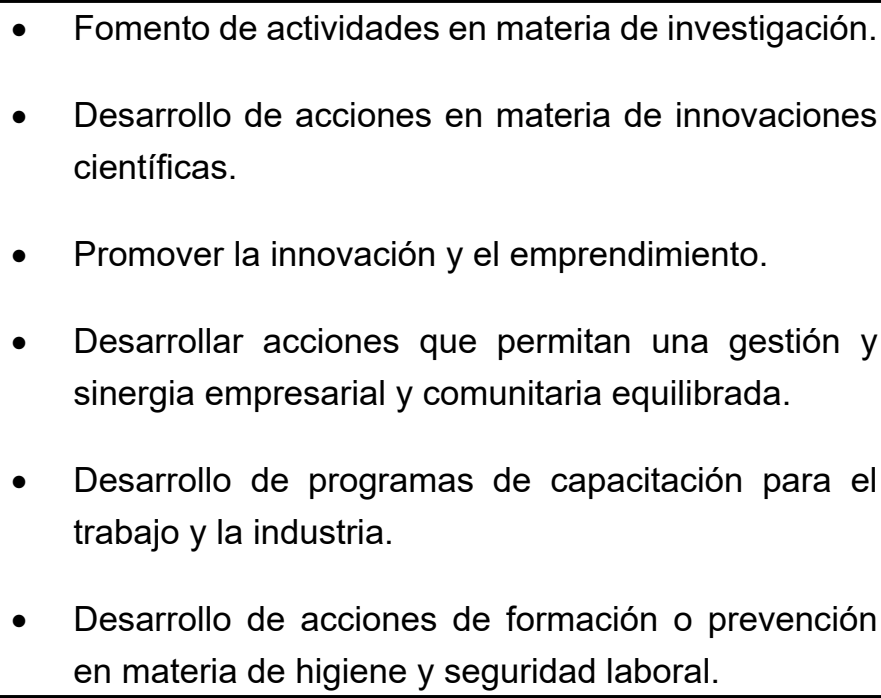 \\
\hline $\begin{array}{l}\text { Objetivo 10: Reducción de las } \\
\text { desigualdades }\end{array}$ & $\begin{array}{l}\text { - Campañas de atención a las poblaciones de } \\
\text { migrantes e inmigrantes. } \\
\text { - Promoción de acciones destinadas al respeto de } \\
\text { las personas indistintamente de su género, etnia, } \\
\text { religión o condición social. } \\
\text { - Fomento de actividades en materia de salud y } \\
\text { educación sobre todo a poblaciones vulnerables. }\end{array}$ \\
\hline
\end{tabular}




\begin{tabular}{|c|c|}
\hline $\begin{array}{l}\text { Objetivo 11: Ciudades y comunidades } \\
\text { sostenibles }\end{array}$ & $\begin{array}{l}\text { - Campañas de seguridad ciudadana. } \\
\text { - Campañas para la conservación y uso adecuado de } \\
\text { los parques, servidumbres y áreas comunes. } \\
\text { - Fomento de actividades para el fortalecimiento de la } \\
\text { mancomunidad. } \\
\text { - Fomento de acciones en materia de educación } \\
\text { comunitaria. }\end{array}$ \\
\hline $\begin{array}{l}\text { Objetivo 12: Producción y consumo } \\
\text { responsable }\end{array}$ & $\begin{array}{l}\text { - Fomento de métodos para la producción y consumo } \\
\text { de bienes y recursos responsables. } \\
\text { - Promoción de huertos en los hogares o de cultivos } \\
\text { hidropónicos. } \\
\text { - Campañas para la eliminación adecuada de los } \\
\text { - Campañas para reducir el desperdicio de alimentos. } \\
\text { - Educación en materia de manejo de presupuestos e } \\
\text { ingresos familiares. }\end{array}$ \\
\hline Objetivo 13: Acción por el clima & $\begin{array}{l}\text { - Campañas de prevención de incendios forestales o } \\
\text { quemas en los hogares. } \\
\text { - Campañas para reducir el consumo de energía } \\
\text { eléctrica, agua y otros elementos que impactan de } \\
\text { manera directa en el clima. } \\
\text { - Fomentar la reforestación y generación de cultivos. } \\
\text { - Campañas para la reducción de contaminantes } \\
\text { generados por las diversas empresas. } \\
\text { Campañas para el uso de transporte público o de } \\
\text { otras formas más amigables con el ambiente afín de } \\
\text { disminuir las emisiones por gases tóxicos generados } \\
\text { por los vehículos. }\end{array}$ \\
\hline
\end{tabular}




\begin{tabular}{|c|c|}
\hline Objetivo 14: Vida submarina & $\begin{array}{l}\text { - Campañas para la conservación y cuido de la vida } \\
\text { submarina. } \\
\text { - Fomento de una pesca responsable en las } \\
\text { comunidades. } \\
\text { - Fomento de acciones para la conservación de } \\
\text { humedales, arrecifes y manglares. } \\
\text { - Campañas para reducir la contaminación de los ríos, } \\
\text { playas y mares que afecta la vida submarina. } \\
\text { - Fomentar acciones destinadas a un uso sostenible } \\
\text { de los recursos oceánicos. }\end{array}$ \\
\hline Objetivo 15: Vida de ecosistemas terrestre & 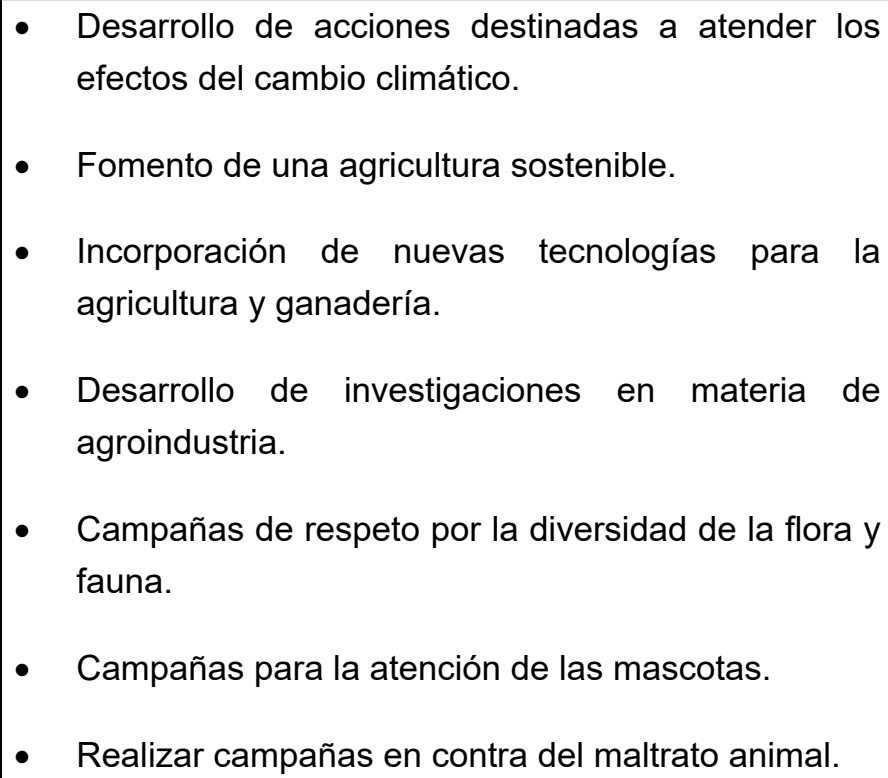 \\
\hline $\begin{array}{l}\text { Objetivo 16: Paz, justicia e instituciones } \\
\text { fuertes }\end{array}$ & $\begin{array}{l}\text { - Fomento de acciones para la promoción de la paz y } \\
\text { los derechos humanos. } \\
\text { - Acciones destinadas a la prevención o mediación de } \\
\text { conflictos, violencia a nivel comunitario o violencia } \\
\text { doméstica. } \\
\text { - Programas destinados a minimizar los delitos a nivel } \\
\text { comunitario. } \\
\text { - Promoción de una educación ciudadana y cívica. } \\
\text { Programas de intervención y rescate social a } \\
\text { - Promoción de acciones de respeto por la vida o por } \\
\text { las personas. }\end{array}$ \\
\hline
\end{tabular}




\begin{tabular}{|c|c|}
\hline $\begin{array}{l}\text { Objetivo 17: Alianza para lograr los } \\
\text { objetivos }\end{array}$ & $\begin{array}{l}\text { - Establecer alianzas estratégicas con organizaciones, } \\
\text { empresas o comunidades para el desarrollo de } \\
\text { proyecto, campañas o programas. } \\
\text { - Vincular tanto a profesores, administrativos como } \\
\text { estudiantes a las diversas actividades destinadas al } \\
\text { logro de los objetivos de desarrollo sostenible, } \\
\text { - Desarrollar diversas acciones dentro del marco del } \\
\text { mes de los objetivos de desarrollo sostenible. } \\
\text { Establecer convenios o acuerdos con entidades } \\
\text { nacionales o internacionales que coadyuven al } \\
\text { desarrollo de los objetivos. }\end{array}$ \\
\hline
\end{tabular}

Fuente: elaborado en base a los resultados del estudio focal y la revisión documental.

\section{DISCUSIÓN Y CONCLUSIONES}

Este estudio, realizado con una población representativa de las diversas provincias más pobladas del país, permitió conocer de primera mano por parte de los principales actores, cuáles eran las necesidades más apremiantes que ellos padecen y que en conjunto con la universidad, pudiesen ser atendidas desde la perspectiva de los diversos factores.

Con los resultados de este estudio, se han podido materializar acciones que impactan en materia de extensión y academia, por tal razón, en casi 2 años, se han gestados hasta la fecha 12 proyectos, 4 campañas, 6 talleres, 2 cursos, 1 foro y 1 diplomado por parte de la propia universidad, que, al salir de la necesidad de las propias comunidades, ha servido de base para disminuir las brechas sociales y económicas, entre estas actividades, podemos mencionar: programas como: Santander verde, proyecto sobre el uso eficiente y racional del agua y la energía, campaña de hábitos saludables, taller de liderazgo para jóvenes, curso de Excel, foro de alimentación saludable y el diplomado de estilo de vida digital, entre otros.

A su vez, se logró la consecución de 16 trabajos comunitarios por parte de los estudiantes a nivel de posgrado, que han tomado como base el estudio realizado, lo que ha significado un gran aporte, porque no sólo se cumple con la meta país de coadyuvar en la gestión de los Objetivos de Desarrollo Sostenible, sino que a su vez, es mucho más vinculante el trabajo realizado por los estudiantes, dado que la comunidad se siente tomada en cuenta, escuchada y permite sobre todo su participación en la elaboración de herramientas para la mejora de la calidad de vida, para lo cual, se enuncian algunos ejemplos de los trabajos comunitarios desarrollados por los estudiantes: gestión y manejo de desechos con innovación social, conocimiento y prevalencia del abuso de esteroides anabólicos androgénicos, promoción de educación emocional en niños de edad escolar, entre otros.

Aún falta mucho por hacer, no obstante, la Universidad Santander ha dado un paso importante en esta vía beneficiando a aproximadamente 1,200 personas, lo que sirve de ejemplo para otras organizaciones que deseen sumarse en lograr una adecuada transformación educativa que permita la erradicación de las barreras sociales y económicas que afectan a los sectores más vulnerables. 


\section{REFERENCIAS BIBLIOGRÁFICAS}

Arauz Quintero, R. R. (18 de enero de 2013). El consumo responsable sí impacta. La Estrella de Panamá.

Asamblea Nacional. (26 de junio de 2015). CONEAUPA. Obtenido de http://www. coneaupa.edu.pa/sites/default/files/ LEY\%2052\%20GO\%2027813-B\%20. pdf

Beluche, O. (31 de octubre de 2018). resumen latinoamericano. Obtenido de http:// www.resumenlatinoamericano. org/2018/10/31/panama-el-reino-de-ladesigualdad-social/

Bernal, C. (2010). Metodología de la investigación. Colombia: Pearson Educación.

Comisión Económica para América Latina y el Caribe (CEPAL) - Naciones Unidas. (mayo de 2016). Sela. Obtenido de http://www.sela.org/media/2262361/ agenda-2030-y-los-objetivos-dedesarrollo-sostenible.pdf

Del Moral, T. (26 de marzo de 2016). Los servicios de los ecosistemas de bosques. La Prensa.

Delgado-Diamante, D. (29 de marzo de 2019). Desigualdad social: las dos caras de Panamá. La estrella de Panamá.

Garrido, E. (13 de junio de 2018). Panamá se ubica tercera en el ranking de ciudades más sostenibles de Latinoamérica 2018. El Capital Financiero.

Gobierno de la República de Panamá. (Julio de 2017). MIDES. Obtenido de https:// www.mides.gob.pa/wp-content/ uploads/2017/09/Informe-Voluntario-
ODS-2017.pdf

Gómez Luna, L. (2002). EcuRed. Obtenido de https://www.ecured.cu/Comunidad

Hernández Sampieri, R., Fernández Collado, C., \& Baptista Lucio, P. (2014). Metodología de la investigación. México: McGrawHill.

Ministerio de Desarrollo Social. (29 de Junio de 2018). INFOJURIDICA. Obtenido de http://gacetas.procuraduria-admon.gob. pa/28558-A_2018.pdf

Ministerio de Educación. (30 de Agosto de 2018). CONEAUPA. Obtenido de http:// www.coneaupa.edu.pa/sites/default/ files/2018-08/decretos $\% 202018 /$ Decreto\%20Ejecutivo\%20539-d.pdf

Ministerio de Relaciones Exteriores. (14 de Septiembre de 2015). INFOJURIDICA. Obtenido de http://gacetas. procuraduriaadmon.gob.pa/27870-B_2015.pdf

Organización de las Naciones Unidas. (2 de septiembre de 2015). un.org. Obtenido de https://undocs.org/es/A/70/1

Programa de las Naciones Unidas para el Desarrollo. (2016). PNUD. Obtenido de https://www.undp.org/content/undp/es/ home/sustainable-development-goals. html

Salazar Atencio, R. E. (1 de febrero de 2018). Energía limpia vs subsidios. La Prensa.

Secretaría Técnica del Gabinete Social. (Agosto de 2015). Despacho de la Primera Dama. Obtenido de https:// www.despachoprimeradama.gob.pa/ tmp/file/509/ODM-\%20Reporte\%20 Actualizado.pdf

Sifuentes, A., Benavides, S., \& Reinozo, M. 
(Julio - Diciembre 2011). El proceso de extensión universitaria: Un análisis desde la perspectiva teórica. Actualidad Contable FACES Año $14 N^{\circ}$ 23, 118133.

Torres, Y., \& Solano, L. (2017). Estudio de las necesidades de extensión y servicios a la sociedad. Panamá: Universidad Santander.

Universidad Santander. (2018). Obtenido de http://www.usantander.edu.pa/ institucional/index.html 